\title{
Population trapping and inversion in ultracold Fermi gases by excitation of the optical lattice - Non-equilibrium Floquet-Keldysh description
}

\author{
Regine Frank \\ Insitute for Solid State Physics, Karlsruhe Institute of Technology (KIT), Wolfgang-Gaede-Strasse 1, 76131 Karlsruhe \\ present address: Institute for Theoretical Physics, Eberhard-Karls Universität Tübingen, Auf der Morgenstelle 14, 72076 \\ Tübingen, Germany
}

Received: 17.12.2012 / Revised version: date

\begin{abstract}
A gas of ultracold interacting quantum degenerate Fermions is considered in a three dimensional optical lattice which is externally modulated in the frequency and the amplitude. This theoretical study utilizes the Keldysh formalism to account for the system being out of thermodynamical equilibrium. A dynamical mean field theory, extended to non-equilibrium, is presented to calculate characteristic quantities such as the local density of states and the non-equilibrium distribution function. A dynamics Franz-Keldysh splitting is found which accounts for the non-equilibrium modification of the underlying bandstructure. The found characteristic Floquet-fan like bandstructure accounts for the quantized nature of the effect over all frequency space.
\end{abstract}

\section{Introduction}

Non-equilibrium physics is often counter-intuitive at first instance and additionally has often been neglected due to the sophisticated methods needed in order to theoretically describe such phenomena. This is even more unfortunate, given that non-equilibrium surrounds us every day in real life, e.g. in hydrodynamic flows etc. Let's start with some simple thoughts to explain the formalism: If we consider as 'toy-model' e.g. a simple two body problem, it is well known that one can transform the whole system in a center-of-mass problem, and a problem which refers to the relative coordinates with respect to that center-of-mass. So far everything is straightforward. If one considers equilibrium physics in the interaction of the two bodies, all processes can be described in terms of the relative coordinates. The center of mass is resting in space and time, or it is moving uniformly. When we consider non-equilibrium processes, the situation changes significantly. The center-of-mass may exhibit a distinctly different behavior in driven, i.e. non- equilibrium, systems. Furthermore, the number of equations or sets of equations required to adequately describe a given system, is usually increased. Partially and among other reasons this is also due to account for the dynamics of the center-of-mass. Because of the center-of-mass dynamics, there may be a significant amount of energy 'stored' or contained in these dynamics. That amount of stored energy consequently does not affect the two body body system directly, to be more precise an imaginary observer on either of the two bodies will not 'see' the results due to the center-of-mass dynamics as he is affected by the dynamics of relative coordinates. In this imaginary setup, the outer world is however also sensitive to the center-of-mass dynamics, which may change the system behavior significantly, as compared to systems without such center-of-mass dynamics.

When we consider ultracold gases, as investigated in various exciting experiments $[1,2,3,4,5,6,7,8$, in optical lattices in non-equilibrium, we take exactly the point of view into account which is described above: The behavior of the atoms in their own frame of reference is not affected by non-equilibrium, they obey the physics with respect to their equilibrium groundstate. Alas the band structure, the dispersion relation which can be measured by the observer from outside is significantly changed. The system all together in non-equilibrium takes a new groundstate as reference, and the equilibrium ground state becomes irrelevant. In consequence, that fact leads e.g. to a significant aberrance of the ground state from the Fermi edge for ultracold Fermions in optical lattices even as a state of half-filling is considered. Furthermore, it is clear that the equilibrium state is not to be reached 'smoothly' from an excited state by decreasing the energy, but that process represents, in fact, a phase transition.

Within this article an ultracold fermionic manyparticle quantum system on a lattice is studied. The lattice is 
modulated by vibrations and the dynamical response is calculated by raise of the modulations' amplitude for various frequencies. This systems are the most ideal setups to study Hubbard physics in non-equilibrium setups.

\section{Model and Theory}

The physical system under consideration is comprised of an optical lattice in three dimensions (3D) in which an ultracold atomic gas has been loaded. The gas consists of fermionic atoms with a strong repulsive on-site interaction. Due to the interaction a strongly correlated system is formed. The $3 \mathrm{D}$ optical lattice, formed by pairs of counter-propagating laser beams, is externally tuned such that the potential strength of the formed lattice changes in a highly controllable way. Consequently, the intrinsic properties of the lattice, such as hopping amplitude or on-site energy, change accordingly. The modulation of the lattice shall be oscillatory in time but otherwise constant. This means the potential depth experienced by an atom at a given site oscillates around fixed value.

The considered system of ultracold fermions placed in an optical lattice with temporal modulations can be described by the following Hamiltonian

$$
\begin{aligned}
& H(\tau)=\sum_{i, \sigma}\left(\epsilon_{0}+\widetilde{E}(\tau)\right) c_{i, \sigma}^{\dagger} c_{i, \sigma} \\
& -\sum_{\langle i, j\rangle, \sigma}(t+\widetilde{T}(\tau)) c_{i, \sigma}^{\dagger} c_{j, \sigma}+\frac{U}{2} \sum_{i, \sigma} c_{i, \sigma}^{\dagger} c_{i, \sigma} c_{i,-\sigma}^{\dagger} c_{i,-\sigma}
\end{aligned}
$$

where the first term on the right hand side (rhs) represents the on-site energy of the atoms, the second term on the rhs the kinetic or hopping contribution between nearest neighbor lattice sites and the last term on rhs represents the repulsive interaction with strength $U$ between two atoms located at the same lattice site. The letter $\tau$ marks the temporal dependence on time, $\epsilon_{0}$ is the equilibrium or static on-site energy, whereas $\widetilde{E}(\tau)$ is the time dependent contribution or modulation of this onsite energy. The hopping in this time dependent model is modified likewise, $t$ is the regular hopping amplitude of an atom between two adjacent lattice sites and $\widetilde{T}(\tau)$ is its time dependent modification. If two atoms reside at the very same lattice site they encounter a repulsive interaction of strength $U$, this interaction strength is however not to be modified by temporal changes due to its overwhelming strength compared to possible temporal changes. The operators $c_{i, \sigma}^{\dagger}$ and $c_{i, \sigma}$ create and annihilate an atom at lattice site $i$ with spin $\sigma$ in Eq. (1). Furthermore, the symbol $\langle i, j\rangle$ refers to a sum over nearest neighbors only.

Throughout this paper, the time dependent modulations of the lattice, and therefore also of the on-site energies and hopping amplitudes, are assumed to be periodic in time. Consequently, the time dependent contributions in Eq. (11) are assumed to be of the form

$$
\begin{aligned}
& \widetilde{E}(\tau)=E \cos (\Omega \tau) \\
& \widetilde{T}(\tau)=T \cos (\Omega \tau)
\end{aligned}
$$

where $\Omega$ represents the frequency which is used to modulate the lattice. A careful choice of parameters $E$ and $T$ guarantee that no sign change as a function of time $\tau$ will occur in the Hamiltonian $H(\tau)$, Eq. (1).

At this point it is worth noting two important points concerning the theory. First, due to the external driving of the optical lattice, the system can not reside in a state of thermodynamical equilibrium and therefore requires special care, intrinsic to the treatment of nonequilibrium systems, as e.g. proposed by Schwinger and Keldysh 9 . In a nonequilibrium system the current state and also physical quantities, such as e.g. the density of states, depend always on two time arguments, for instance a starting time and the elapsed time, or equally a relative time and a center-of-mass time. In contrast, in equilibrium theory, there is usually only one time coordinate needed, the relative time, since the center of mass time can not change any physics. However, in the considered nonequilibrium system, physical quantities depend on two time arguments. An appropriate formalism to consistently describe such systems using e.g. diagrammatic descriptions, has been developed first by Schwinger and then by Keldysh [9], by introducing a Green's function according to

$$
G\left(\tau_{1}, \tau_{2}\right)=\left(\begin{array}{ll}
G^{++}\left(\tau_{1}, \tau_{2}\right) & G^{+-}\left(\tau_{1}, \tau_{2}\right) \\
G^{-+}\left(\tau_{1}, \tau_{2}\right) & G^{--}\left(\tau_{1}, \tau_{2}\right)
\end{array}\right)
$$

where the superscripts depend on which branch of the Schwinger-Keldysh-Schwinger contour the time arguments reside. To give an explicit example, $G^{++}\left(\tau_{1}, \tau_{2}\right)$ refers to a situation, where $\tau_{1}$ and $\tau_{2}$ are both the upper $(+)$ countour path. By using a rotation $R$, given by

$$
R=\frac{1}{\sqrt{2}}\left(\begin{array}{rr}
1 & -1 \\
1 & 1
\end{array}\right),
$$

in this Keldysh space, the Keldysh Schwinger Green's function may also be written in the form

$$
G\left(\tau_{1}, \tau_{2}\right)=\left(\begin{array}{ll}
0 & G^{a d v}\left(\tau_{1}, \tau_{2}\right) \\
G^{\text {ret }}\left(\tau_{1}, \tau_{2}\right) & G^{\text {keld }}\left(\tau_{1}, \tau_{2}\right)
\end{array}\right)
$$

where $G^{a d v}$ and $G^{r e t}$ are the well known advanced and retarded components of the Green's function, and the new $G^{\text {keld }}\left(\tau_{1}, \tau_{2}\right)$ represents the so-called Keldysh component of the Green's function. As in equilibrium physics, the advanced and retarded parts are connected to the actual state of the system, i.e. its spectral weight and the density of states, whereas the Keldysh component additionally includes information on the nonequilibrium distribution. Especially the latter point will utilized later on to find the according non-equilibrium distribution 
function. The second point to note here is the strictly periodic nature of the modulation, which allows for the use of the so-called Floquet theory [10. In order to take full advantage of these conditions, it is appropriate to change to Fourier space and using a momentum and frequency description. As detailed above, the description of nonequilibrium systems requires the use of two independent time arguments and consequently requires a two-time Fourier transform towards the corresponding two-frequency expression. The relative frequency $\omega$ is the very same as known from equilibrium theory and the center of mass frequency corresponds to the modulation frequency of the optical lattice $\Omega$. In particular, the employed Fourier transform reads

$$
\begin{aligned}
G_{m n}^{\alpha \beta}(k, \omega, \Omega)= & \int_{-\infty}^{+\infty} \mathrm{d} \tau_{r e l} \frac{1}{\mathcal{T}} \int_{-\mathcal{T} / 2}^{+\mathcal{T} / 2} \mathrm{~d} \tau_{c m} e^{i\left(\omega \frac{m+n}{2} \Omega_{L}\right) \tau_{r e l}} \\
& \times e^{i(m n) \Omega_{L} \tau_{c m}} G^{\alpha \beta}\left(k, \tau_{r e l}, \tau_{c m}\right)
\end{aligned}
$$

where the Greek superscripts denote the Keldysh indices, i.e. $\alpha$ and $\beta$ may assume the values + or - depending on which branch of the Keldysh contour the time arguments reside. The subscripts $m$ and $n$ label the Floquet index, i.e. they account for the number of absorbed or emitted lattice quanta $\hbar \Omega$. The quantity $\mathcal{T}$ in Eq. (7) is the time period $\mathcal{T}=\frac{2 \pi}{\Omega}$, the system time is shifted to a center of motion time $\tau_{c m}=\frac{\tau_{1}+\tau_{2}}{2}$ and a relative time coordinate $\tau_{r e l}=\tau_{1}-\tau_{2}$. The resulting Keldysh Schwinger Floquet Green's function is therefore a matrix Green's function of dimension $2 \times 2$ in Keldysh space and of dimensions $(2 n+1) \times(2 m+1)$ in Floquet space. In numerical evaluations, a size of $21 \times 21$ has been used in Floquet space, corresponding to a consideration of emission and absorption of up to 10 lattice quanta at the same time (10 phonon processes). Combinations of these techniques Schwinger-Keldysh technique with the Floquet formalism have been considered for some time see e.g. [13, 14, 15.

The Hamiltonian, Eq. (1), including the finite on-site interactions, is solved at zero temperature and at half filling of the lattice sites by extending an equilibrium dynamical mean field theory (DMFT), see for instance reference [11, towards accounting for this nonequilibrium situation by including the Floquet-Keldysh Greens function described in Eq. (7). This DMFT maps the nonequilibrium interacting lattice system onto a local impurity system, still out of thermal equilibrium, by assuming a local on-site selfenergy. This is in some way comparable to the light-matter interaction considered in ref. [14. The DMFT provides a solution for the derived local impurity problem. The iterated perturbation theory (IPT) 12 has been extended to a nonequilibrium description. This method as a diagrammatic impurity solver is reasonable fast also for nonequilibrium systems, because there exist analogues [9] to the Feynman rules for evaluating equilibrium diagrams. Especially at half filling,

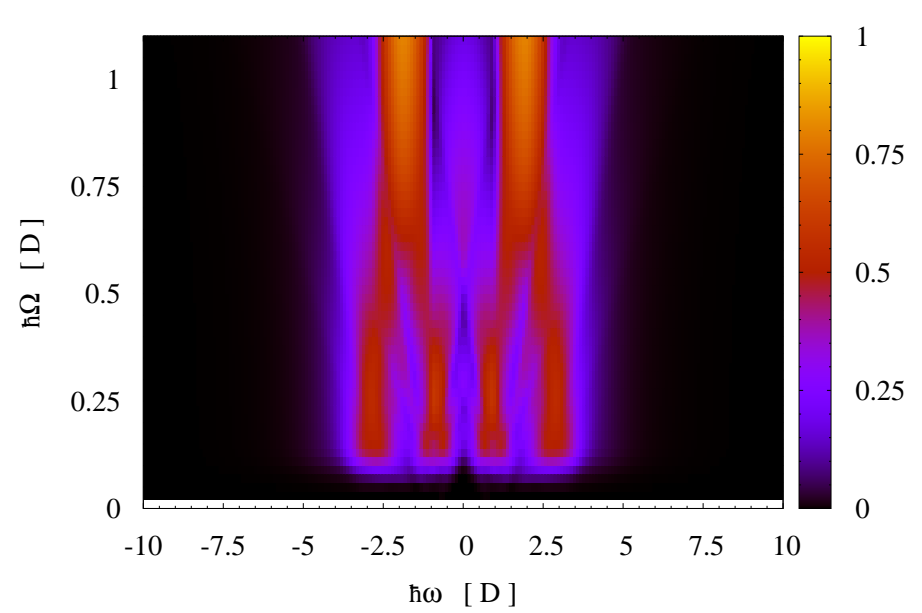

Fig. 1 Imaginary part of the retarded component of the full and local Green's function. This relates to the density of states (LDOS) $N(\omega, \Omega)$, as given in Eq. (8) . The abscissa represents the atomic energy $\hbar \omega$, whereas the ordinate $\hbar \Omega$ labels the energy of the lattice modulation, i.e. its frequency. Here and in the following the unit of energy is given by the half bandwidth $D$ of the equilibrium system. The interaction strength of two atoms with opposite spin at the same lattice site is $U / D=4$ and the modulation hopping amplitude is here set to be $T / D=0.25$. For a detailed discussion see text.

the IPT proves to be a reliable solver for the DMFT in equilibrium systems [12].

At the end one is left with the task of selfconsistently evaluating a matrix equation which is of dimensions $2 \times 2$ in Keldysh space and of dimension $(2 n+1) \times(2 n+1)$ in Floquet space. In this paper $n=10$ Floquet bands have been used in the numerical evaluations, i.e. $n$ ranges from -10 to +10 . Practically, this number of Floquet bands corresponds to the number of emitted or absorbed lattice quanta $\hbar \Omega$. The numerical solution of this nonequilibrium DMFT approach, leads then to the full nonequilibrium Floquet-Keldysh-Greens function Eq. (77), i.e. to the full knowledge of the three distinct components of the local Green's function $G^{r e t}(\omega, \Omega), G^{r a d v}(\omega, \Omega)$ and as well as $G^{k e l d}(\omega, \Omega)$ for all atomic energies $\hbar \omega$ and all lattice vibrations $\hbar \Omega$. Therefore, also to physical quantities such as the local density of states or the nonequilibrium distribution function of the ultracold fermionic gas in the modulated optical lattice. In particular the local density of states $N(\omega, \Omega)$ is given by the expression

$$
\begin{aligned}
N(\omega, \Omega) & =-\frac{1}{\pi} \operatorname{Im} G^{r e t}(\omega, \Omega) \\
& =-\frac{1}{\pi} \int \mathrm{d}^{3} k \sum_{m, n, \sigma} \operatorname{Im} G_{m n ; \sigma}^{r e t}(k, \omega, \Omega)
\end{aligned}
$$

since all emission and absorption processes have to be included, there appears a sum over all involved Floquet bands $\left(\sum_{m n} \ldots\right)$ in Eq. (8). 


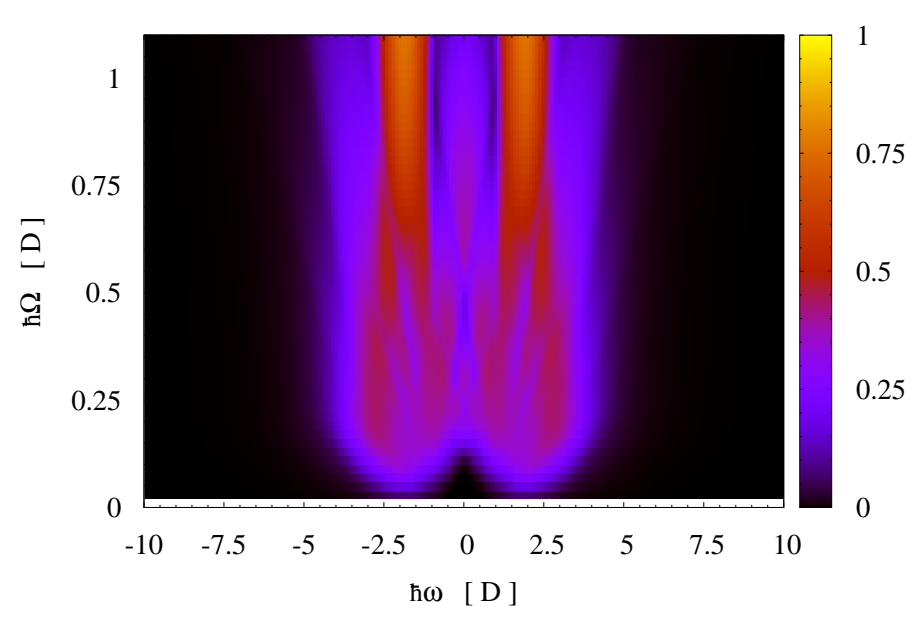

Fig. 2 The same parameters as in Fig. 1 but with the modulation induced hopping increased to a value of $T / D=1.00$. For a detailed discussion see text below.

\section{Results and Discussion}

In this section results of the numerical evaluation of the nonequilibrium DMFT are presented for a fixed set of on-site energy and on-site interaction strength and for a range of modulation strengths $T$ of the optical lattice. The on-site energy variation is here set to be $E / D=1$. Here throughout this paper the unit of energy is given by the half bandwidth $D$ of the equilibrium system. In particular, the system is at half filling, the on-site interaction is set to be $U / D=4$, i.e. it is a strongly interacting system. The equilibrium ground state of this system is therefore an insulating state, i.e. there is no spectral weight at the Fermi energy $\hbar \omega=0$. The two bands, i.e. the lower and the upper Hubbard band, are split by $U / 2$ due to the strong correlations. In other words, a gap of width $U / 2$ opens up in the spectral weight, consequently conductivity is suppressed and a Mott insulating state is established. The energy gap occurs symmetrically in the presented plots, because in the calculations the filling of the optical lattice is assumed to be 0.5 , i.e. each lattice site is occupied by one atom. In the presence of periodic lattice strength variations, i.e. in the non-equilibrium regime, this situation is altered. With increasing strength of the lattice modulations and depending on the modulation frequency $\Omega$ this changes in some respects, as detailed below.

In Figs. 1 to 7 the local density of states is displayed for a series of increasing amplitudes of the periodic optical lattice modulation. Each plot itself features the atomic energy $\hbar \omega$ along the abscissa and the energy $\hbar \Omega$, i.e. frequency, of the lattice modulations along the ordinate. So, for a specified lattice vibration frequency, one chooses that specific value at the y-axes and reads out the density of states as a function of atomic energy $\hbar \omega$ along the $\mathrm{x}$-axes.

For small external modulation frequencies the original bands are found to split into a number of Floquet-

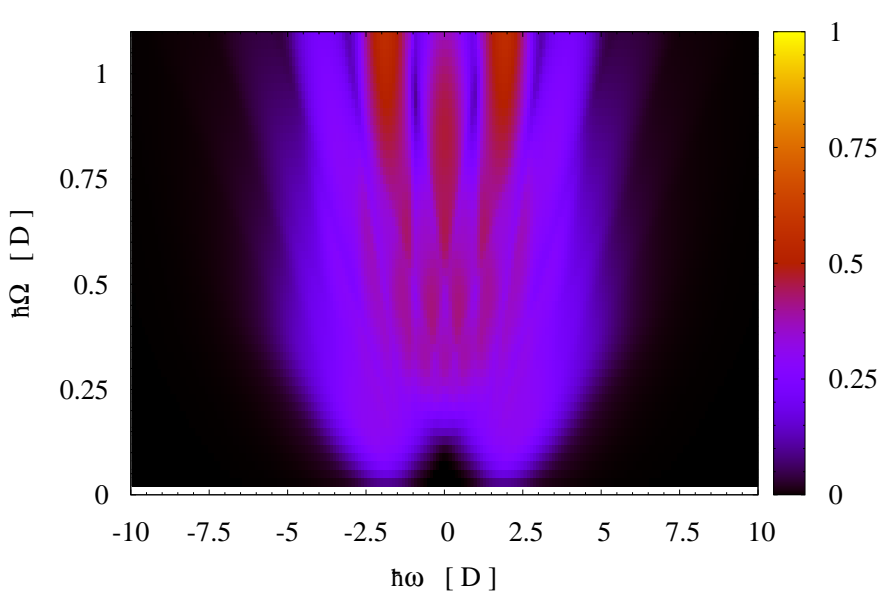

Fig. 3 The same parameters as in Fig. 1 but with the modulation induced hopping increased to a value of $T / D=3.00$. For a discussion see text.

sidebands which are separated to each other proportional to the external modulation frequency, therefore the almost equally smeared-out appearance for small vibrational frequencies, see e.g. Fig. 1, For this example of a small amplitude modulation $T / D=0.25$, another interesting effect is to be observed, starting at about modulation energies of $\hbar \Omega / D=0.12$.

The interaction-based splitting of the bandstructure of upper and lower Hubbard band in Fig. 1 is modified in the way that the two Hubbard bands themselves are again split into two, such that an effective four-band system is formed. This additional splitting is however not caused by atom-atom interactions but by the dynamical modulation of the lattice potential. This newly established state still maintains, at least approximately, the insulating character of the underlying equilibrium solution, i.e. no spectral weight and atomic occupation at the Fermi edge, i.e. at $\hbar \omega=0$. These four formed quasibands are separated by regions of low spectral weight, so to speak by quasi-gaps which opened solely due to externally induced lattice vibrations in this ultracold Fermigas system. In a modulation range between $\hbar \Omega / D=0.4$ and $\hbar \Omega / D=0.6$ where the fast modulations of the lattice only weakly affect the local density of states of the degenerate atomic gas.

The decreasing effect of the dynamic lattice vibrations on the density of states with increasing frequencies $\Omega$ is attributed to the fact that at some point the atoms in the optical lattice cannot follow the quickly changing lattice potential anymore. Therefore, a local density of states is formed which is in its main features similar to the equilibrium density of states, compare to Fig. 1 and vibration frequencies of ca. $\hbar \Omega / D \geq 1$. In this regime, only one excited Floquet band of both upper and lower Hubbard band is still visible, with minor deviations inside the equilibrium gap, i.e. between ca. $-1<\hbar \omega / D<+1$. Each Hubbard band forms a Floquet sideband of emission of one vibrational quant $\hbar \Omega$ 


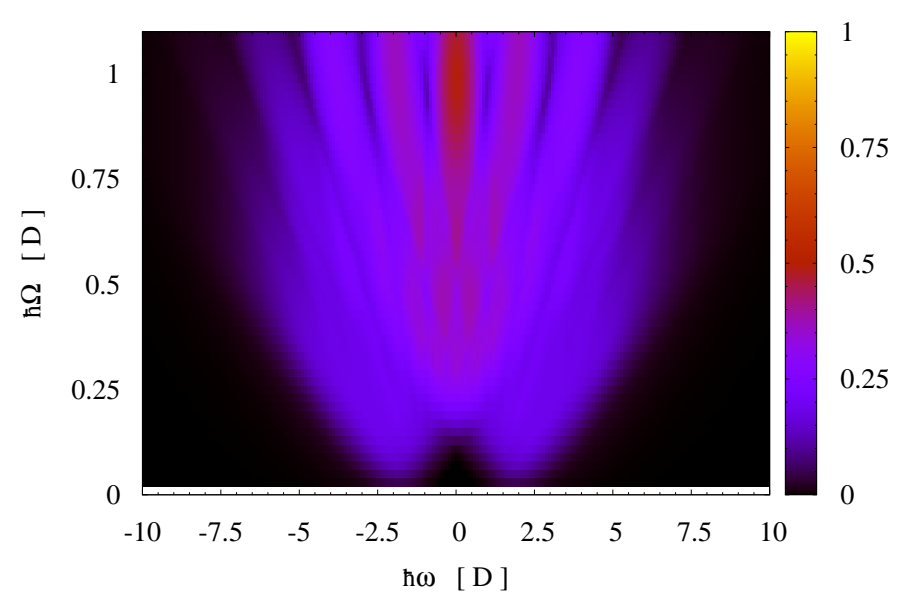

Fig. 4 The same parameters as in Fig. 1 but with the modulation induced hopping increased to a value of $T / D=5.00$. For a detailed discussion see text below.

to its left and one Floquet sideband of absorption of one vibrational quant $\hbar \Omega$ to its right hand side.

In Figs. 2 15, we present numerical results for the LDOS, Eq. (8), for numerically ascending modulation induced hopping strengths of $T / D=1,3,5,8$. The spectral weight for intermediate strengths $T / D$, Fig. 2 and Fig. 3. the range of small modulation energies $\hbar \Omega \leq$ 0.25 , displays the change from modified equilibrium DOS towards a split up two band system, split into a quite large number of Floquet side bands. The synthetic, generated quasi four-band system is still found for approximately $\hbar \Omega / D \leq 0.65$. For even larger modulation energies in Fig. 2 and Fig. 3 the spectral weight assembles again the equilibrium two Hubbard bands and to some extent an additional spectral weight in the gap region between $1 \leq \hbar \omega / D \leq 1$. For quite large hopping modulations of $T / D=5$ and $T / D=8$ presented in Fig. 4 and Fig. 5, the just described characteristics are absent and replaced by a Floquet-fan like structure. This found structure represents the Floquet sidebands of absorption and emission of vibration quanta of the lattice. Interesting to note about Fig. 4 and Fig. 5 is that the structure of the LDOS for large $\hbar \Omega$ displays a reversed structure as compared to the equilibrium system. Precisely, in the former gap region $1 \leq \hbar \omega / D \leq 1$, there is significant spectral weight, whereas in the place of the former Hubbard bands the spectral weight is suppressed.

In general, this formed structure in the spectral function resembles a Wannier-Stark ladder, which is also seen in the energy distribution of the fermionic atoms in the lattice. The non-equilibrium distribution function $F(\omega, \Omega)$ of the ultra-cold and strongly interacting Fermi gas is obtained with the help of the Keldysh component $G^{\text {Keld }}(\omega, \Omega)$ of the full Keldysh-Floquet-Green's function by means of the following general relation

$$
F(\omega, \Omega)=\frac{1}{2}\left(1+\frac{1}{2 i} \frac{\sum_{m n} G_{m n}^{K e l d}(\omega, \Omega)}{\sum_{m n} \operatorname{Im} G_{m n}^{r e t}(\omega, \Omega)}\right)
$$

A numerical evaluation of this distribution function, characterizing the atoms inside the periodically modulated lattice, is depicted in Fig. 6. The distribution function $F(\omega, \Omega)$ is depicted for various modulations strengths $T$ as a function of atomic energy $\hbar \omega$ for one specific value of the modulation frequency $\hbar \Omega / D=1$. Clearly visible are the absorption and emission features of lattice modulation quanta, a spectral hole burning effect. The absorption features for $\hbar \omega / D<0$ display are sharp lower edge, since there is a specific maximum energy for each absorption process.

The different absorption features correspond to the different number of involved lattice quanta. For instance, atoms that absorb energy at $-\hbar \omega$ are lifted in energy to a state with energy $+\hbar \omega$ by absorbing energy from the lattice oscillation with $2 \hbar \omega=n \hbar \Omega$ where $\mathrm{n}$ characterizes the number of absorbed lattice quanta. Processes involving simultaneous absorption of up to four lattice quanta are found. The process of transferring energy to the lattice by emitting lattice quanta is analog. In Fig. 6 the at first glance overproportional dip (peak) in the distribution function at atomic energies $\hbar \omega / D \sim-8$ $(\hbar \omega / D \sim+8)$ is best understood when bearing in mind that the physical quantity involved here is the atomic occupation number, which is the product of distribution and spectral function. Since this effect in the distribution function occurs in a region where the spectral function, see for instance Fig 4, is weak, the occupation number is only slightly modified by this process.

Returning now to the discussion of the density of states. With increasing modulation strength $T$ of the optical lattice holding the ultracold atomic gas, the distortion of the equilibrium density of states becomes more and more severe. By comparing Figs. 2, 4 one observes that the equilibrium density of states is almost completely changed. This is due to the formation of an increasing number of Floquet sidebands. These absorption and emission bands may also encounter intersections and fill even the Hubbard gap. This transferring of spectral weight towards atomic energies $-1<\hbar \omega / D<1$ leads to the breakdown of the Mott insulating state and consequently the system selfconsistently passes over into a conducting state.

With an even more increased modulation amplitude $T$, see e.g. Fig. 5, the system undergoes a transition into a state in which for rather large modulation frequencies ca. $\hbar \Omega / D>0.8$ the system almost resembles a seven-band system with the zeroth band right around the Fermi-energy $\hbar \omega / D=0$, even though the system is severely driven out of equilibrium. The number and position of the Floquet sidebands is also highly sensitive to driving frequency and may change drastically, depending on the specific value of $\hbar \Omega / D$, cf. Fig. 5. Interestingly, this behavior opens a way to switch the system in a controlled way from an insulating multi-band system to a conducting multi-band system, by changing the modu- 


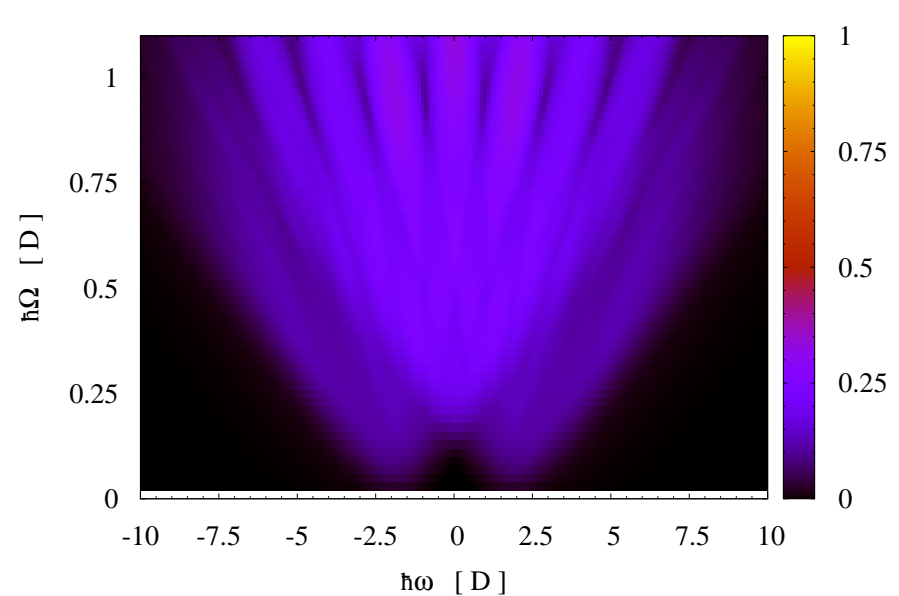

Fig. 5 The same parameters as in Fig. 1 but with the modulation induced hopping increased to a value of $T / D=8.00$. For a detailed discussion see text below.

lation amplitudes from low to high at a fixed external modulation frequency.

The density of states at the Fermi energy is always of special interest, since it is a reliable indicator for the conductivity of the system in question. In Fig. 7 the local density of states at the Fermi edge is depicted for a variety of modulation strengths. Different curves correspond to different modulation strengths as indicated. These curves therefore correspond to vertical cuts along $\hbar \omega / D=0$ in Figs. 115 and are presented in their own graph simply for the reasons of clarification and clearness. Strong non-monotonic variations of the spectral weight are observed depending on both, the modulation strength and frequency. As universal features, there appears a minimum at ca. $\hbar \Omega / D=0.5$ followed by a maximum at ca. $\hbar \Omega / D=0.65$. For small and large modulation frequencies the spectral weight returns to zero, which is its equilibrium value, since the corresponding ground state is a Mott insulator. In general, two-phonon processes which are bridging the gap at modulation energies of $\hbar \Omega=1$ yield a strong conductivity in this driven system.

\section{Summary and Conclusion}

In conclusion, an ultracold gas of strongly interacting fermionic atoms placed in a three-dimensional optical lattice has been considered. The potential strength of the optical lattice has been periodically modulated in time. Due to these external modulations the gas is driven out of thermodynamical equilibrium. A theoretical analysis of the nonequilibrium state was performed by employing the Schwinger-Keldysh technique in order to account for far off equilibrium situation and in combination with a Floquet analysis, best suited to take advantage of the periodic nature of the driving. The system has been solved by a DMFT method, developed to include the nonequilibrium Floquet-Keldysh formalism and solved by means

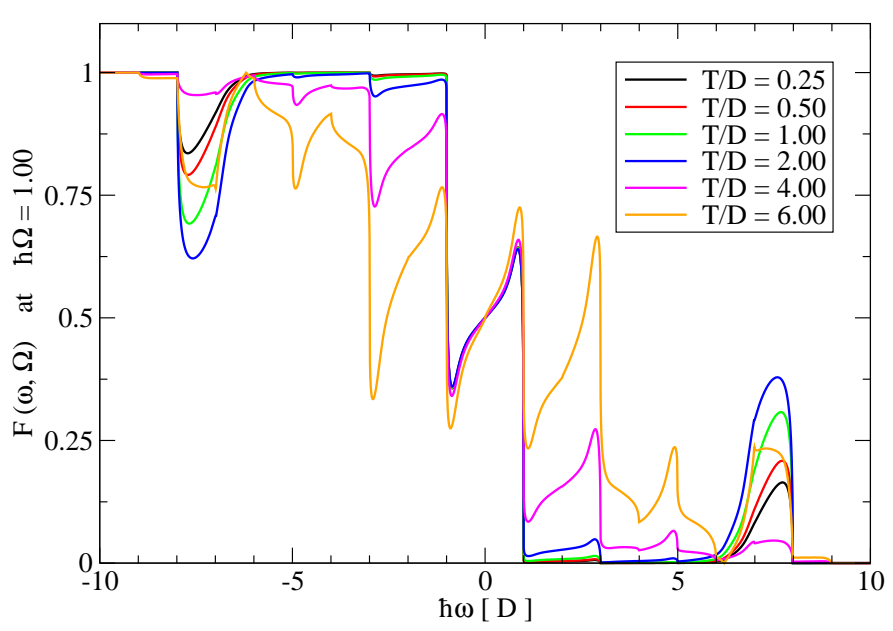

Fig. 6 Numerically computed non-equilibrium distribution function $F(\omega, \Omega)$ of the dynamical AC-Wannier-Stark effect of fermionic atoms as a function of atomic energy $\hbar \omega$ for the modulation frequency of $\hbar \Omega / D=1.0$. Clearly visible are the absorption and emission features of lattice modulation quanta, a spectral hole burning effect. The absorption features for $\hbar \omega / D<0$ display are sharp lower edge, since there is a specific maximum energy for each absorption process. The different absorption features correspond to the different number of involved lattice quanta. Processes involving simultaneous absorption of up to four lattice quanta are found.

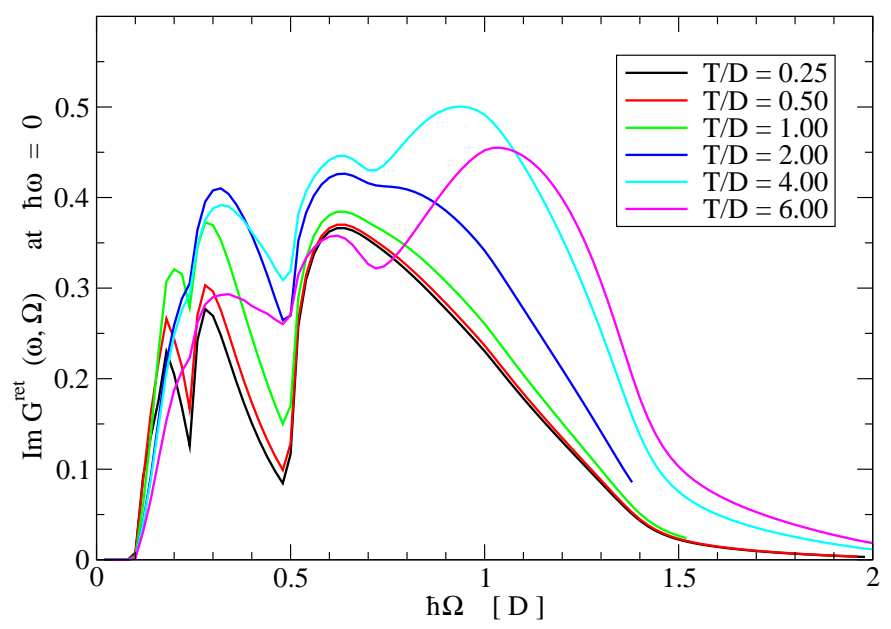

Fig. 7 Displayed is the computed spectral weight $\operatorname{Im} \mathrm{G}^{\text {ret }}(\omega, \Omega)$, c.f. Eq. (8), along the Fermi edge at $\hbar \omega / D=0$ as a function of lattice modulation frequency $\hbar \Omega$. Different curves correspond to different modulation strengths as indicated. The density of states at the Fermi edge is also an indicator of the conductivity of system. Strong non-monotonic variations of the spectral weight are observed depending on both, modulation strength and frequency. As universal features, there appears a minimum at ca. $\hbar \Omega / D=0.5$ followed by a maximum at ca. $\hbar \Omega / D=0.65$. Interestingly, for small and large modulation frequencies the spectral weight returns to zero, its equilibrium value.

of the IPT. The IPT impurity solver has also been extended to account for nonequilibrium system. 
The developed technique was applied to calculate the local density of states of the driven system of an ultracold Fermi-gas in a modulated three-dimensional optical lattice. The influence of a systematic change of the externally controlled amplitude of the lattice modulation was studied. The numerically determined LDOS displays severe changes depending on both the driving frequencies and the modulation amplitude. The strongly correlated system can be driven out of its insulating state into a conducting multi-band dynamical Wannier-Stark state with quite interesting effects in the intermediate regime. The observed inversion as compared to the equilibrium state, i.e. the occupied states above the lower Hubbard band and consequently the lowered population below, is open to experimental verification. In particular the part of the gap region $0 \leq \hbar \omega / D \leq 1$ for large modulations of the lattice. An investigation of this effect towards technological application will be part of future work.

\section{References}

1. W. Ketterle, M. W. Zwierlein, Proceedings of the Internationl School of Physics "Enrico Fermi", Course CLXIV, Varenna, 20 - 30 June 2006, eds. M. Inguscio, W. Ketterle, and C. Salomon (Amsterdam, IOS Press 2008).

2. I. Bloch, J. Dalibard, W. Zwerger, Rev. Mod. Phys., 80, 885 (2008).

3. H. Lignier, C. Sias, D. Ciampini, Y. Singh, A. Zenesini, O. Morsch, E. Arimondo, Phys. Rev. Lett. 99, 220403 (2007).

4. C. Sias, H. Lignier, Y. P. Singh, A. Zenesini, D. Ciampini, O. Morsch, E. Arimondo, Phys. Rev. Lett. 100, 040404 (2008).

5. A. Zenesini, H. Lignier, D. Ciampini, O. Morsch, E. Arimondo, Phys. Rev. Lett. 102, 100403 (2009).

6. J. Struck, C. Ölschläger, R. Le Targat, P. Soltan-Panahi, A. Eckardt, M. Lewenstein, P. Windpassinger, K. Sengstock, Science 333, 996 (2011).

7. J. Struck, C. Ölschläger, M. Weinberg, P. Hauke, J. Simonet, A. Eckardt, M. Lewenstein, K. Sengstock, P. Windpassinger, Phys. Rev. Lett. 108, 225304 (2012).

8. P. Hauke, O. Tieleman, A. Celi, C. Ölschläger, J. Simonet, J. Struck, M. Weinberg, P. Windpassinger, K. Sengstock, M. Lewenstein, A. Eckardt, Phys. Rev. Lett. 109, 145301 (2012).

9. L. V. Keldysh, Sov. Phys. JETP 20, 1018 (1965).

10. M. Grifoni, P. Hänggi, Phys. Rep. 304, 229 (1998).

11. A. Georges et al, Rev. Mod. Phys. 68, 13 (1996).

12. X. Y. Zhang, M. J. Rozenberg, and G. Kotliar, Phys. Rev. Lett. 70, 1666 (1993).

13. A. P. Jauho, K. Johnsen, Phys. Rev. Lett. 76, 4576 (1996).

14. N. Tsuji, T. Oka, H. Aoki, Phys. Rev. B 78, 235124 (2008).

15. J. D. Sau, E. Berg, B. I. Halperin, arxiv 1206.4596 (2012) 